ECCOMAS

Proceedia
UNCECOMP 2021

$4^{\text {th }}$ ECCOMAS Thematic Conference on Uncertainty Quantification in Computational Sciences and Engineering

M. Papadrakakis, V. Papadopoulos, G. Stefanou (eds.) Streamed from Athens, Greece, 28 -30 June 2021

\title{
LIMIT REPRESENTATIONS OF IMPRECISE RANDOM FIELDS
}

\author{
Mona M. Dannert ${ }^{1, *}$, Johannes L. Häufler ${ }^{1}$ and Udo Nackenhorst ${ }^{1}$ \\ ${ }^{1}$ Leibniz University Hannover, Institute of Mechancis and Computational Mechanics \\ Appelstraße 9a, 30167 Hannover \\ *e-mail: mona.dannert@ibnm.uni-hannover.de
}

\begin{abstract}
In order to describe spatially uncertain parameters by random fields, the underlying autocorrelation structure in engineering structures is usually not known.. The idea of imprecise random fields is to acknowledge this lack of knowledge by adding epistemic uncertainties. Within this contribution the influence of the correlation length is studied. In particular, it is shown that there exist bounds that limit the case of having no idea at all. This "absolutely no idea p-box" is defined by white noise and the random variable corresponding to the mean value and standard deviation of the imprecise random field. By this, the limits of having "absolutely no idea" can be described without the need of Karhunen-Loève expansion and random field propagation. Then, at least for linear problems, every response in between can be estimated by linear interpolation without any need for sampling.
\end{abstract}

Keywords: Imprecise random fields, interval valued correlation length, Karhunen-Loève expansion, stochastic finite element method

ISSN:2623-3339 (C) 2021 The Authors. Published by Eccomas Proceedia.

Peer-review under responsibility of the organizing committee of UNCECOMP 2021.

doi: 10.7712/120221.8024.19110 


\section{INTRODUCTION}

Stochastic finite element (FE) method aims to describe a models response depending on random input variables such as loads or material parameters. In this context, uncertainties are distinguished into aleatory and epistemic [5]. The former describe the irreducible, intrinsic randomness of a parameter and is classically described by probability theory. The latter is caused by a lack of knowledge or data. However, gaining enough information to reduce epistemic uncertainty is usually limited by finite resources or limited technical capabilities in engineering reality. To consider such epistemic uncertainties, several possibilistic approaches can be used, e.g. interval [9] or fuzzy [8] analyses. An honest approach usually considers both, aleatory and epistemic uncertainties. Alternative approaches on the treatment of these mixed uncertainties, e.g. evidence theory, fuzzy probabilities or probability boxes, have been reviewed for example in [1].

In probability theory, random fields can be used to describe spatially uncertain values in terms of their mean value, standard deviation and autocorrelation structure. While mean value and standard deviation can be estimated by experiments quite easily, the autocorrelation between the random field values at two different locations can hardly be measured. To describe such mixed aleatory and epistemic uncertainties, imprecise random fields have been introduced lately [6]. This approach can be understood as an extension of probability box (p-box) approach towards random fields. The random field parameters that cannot be determined precisely, e.g. the correlation length, can be described as interval [3] or fuzzy valued [10]. Propagating such imprecise random fields through an FE model, the quantity of interest is described by a p-box, meaning a lower and upper bound instead of a crisp distribution. However, by introducing a second loop over the epistemic uncertainties the sampling process can become very expensive. Discretising the individual random fields for each correlation length by Karhunen-Loève (KL) expansion furthermore leads to high-dimensional problems, especially when small correlation lengths are involved $[3,4]$.

This contribution focuses on the autocorrelation structure where the lack of information is incorporated by an interval valued correlation length. For this purpose, the concept of imprecise random fields and the KL expansion as a method to discretise random fields are introduced in Section 2. It is important to ensure that the imprecise response is not affected by the local or global error arising from the truncation of the KL expansion [4]. Therefore, an imprecise random field input is carefully investigated in Section 3 in terms of the affect of the correlation length and truncation order. Furthermore, the limits of the correlation length towards zero (white noise) and infinity (random variable) are studied. Afterwards, the propagation of imprecise random field input parameters is studied for a linear problem including load and material uncertainties within Section 4. Finally, the results are summarised and concluded in Section 5.

\section{IMPRECISE RANDOM FIELDS}

The concept of imprecise random fields allows to model one or several parameters of a random field by interval or fuzzy variables. This way, epistemic uncertainties can be added to a classically aleatory random field. Mixed uncertainties are usually propagated by a double loop approach. After discretising the epistemic parameters within an outer loop, the probabilistic problem resulting for each crisp parameter can be solved within the inner loop, e.g. by Monte Carlo (MC) sampling. In case of imprecise random fields this means that each resulting random field needs to be discretised. A well known method for this purpose is given by Karhunen-Loève (KL) expansion [7] which will be shortly summarised in the following subsection. Afterwards 
the main idea of the probability box (p-box) approach is illustrated in order to describe and propagate imprecise random fields.

\subsection{Random field discretisation}

Random fields $X(\boldsymbol{z}, \omega)$ are parameters depending on space $\boldsymbol{z} \in \mathcal{D}^{d}$ and chance $\omega \in \Omega$. They can be described in terms of a mean value $\mu_{X}(\boldsymbol{z})$ and an autocovariance function $\operatorname{Cov}\left(\boldsymbol{z}_{1}, \boldsymbol{z}_{2}\right)=$ $\sigma_{X}\left(\boldsymbol{z}_{1}\right) \sigma_{X}\left(\boldsymbol{z}_{2}\right) \Gamma\left(\boldsymbol{z}_{1}, \boldsymbol{z}_{2}\right)$, where $\sigma_{X}(\boldsymbol{z})$ is the standard deviation and $\Gamma\left(\boldsymbol{z}_{1}, \boldsymbol{z}_{2}\right)$ describes the autocorrelation between the random variables assigned to two arbitrary locations $z_{1}$ and $z_{2}$.

Within this contribution, the standard deviation is assumed to be constant, $\sigma_{X}(\boldsymbol{z})=\sigma_{X}$. Then the random field can be described in terms of $\mu_{X}(\boldsymbol{z}), \sigma_{X}$ and $\Gamma_{X}\left(\boldsymbol{z}_{1}, \boldsymbol{z}_{2}\right)$ and its series expansion reads [11]

$$
X(\boldsymbol{z}, \omega)=\mu_{X}(\boldsymbol{z})+\sigma_{X} \sum_{i=1}^{\infty} \sqrt{\lambda_{i}} \phi_{i}(\boldsymbol{z}) \xi_{i}(\omega),
$$

where $\xi_{i}$ are independent standard normal distributed random variables. The eigenpairs $\left\{\lambda_{i}, \phi_{i}\right\}$ are gained by solving

$$
\int_{\mathcal{D}} \boldsymbol{\Gamma}_{X}\left(\boldsymbol{z}_{1}, \boldsymbol{z}_{2}\right) \boldsymbol{\phi}_{i}\left(\boldsymbol{z}_{2}\right) \mathrm{d} \boldsymbol{z}_{2}=\lambda_{i} \boldsymbol{\phi}_{i}\left(\boldsymbol{z}_{1}\right),
$$

where $\Gamma_{X}\left(\boldsymbol{z}_{1}, \boldsymbol{z}_{2}\right)$ can be decomposed as

$$
\boldsymbol{\Gamma}_{X}\left(\boldsymbol{z}_{1}, \boldsymbol{z}_{2}\right)=\sum_{i=1}^{\infty} \lambda_{i} \boldsymbol{\phi}_{i}\left(\boldsymbol{z}_{1}\right) \boldsymbol{\phi}_{i}\left(\boldsymbol{z}_{2}\right) .
$$

To propagate random field parameters through a model, e.g. a stochastic finite element (FE) problem, the infinite sum in Equation (1) needs to be truncated and the random field is approximated by

$$
\hat{X}(\boldsymbol{z}, \omega)=\mu_{X}(\boldsymbol{z})+\sigma_{X} \sum_{i=1}^{T} \sqrt{\lambda_{i}} \phi_{i}(\boldsymbol{z}) \xi_{i}(\omega) .
$$

The corresponding expanded autocorrelation function then reads

$$
\hat{\boldsymbol{\Gamma}}_{X}\left(\boldsymbol{z}_{1}, \boldsymbol{z}_{2}\right)=\sum_{i=1}^{T} \lambda_{i} \boldsymbol{\phi}_{i}\left(\boldsymbol{z}_{1}\right) \boldsymbol{\phi}_{i}\left(\boldsymbol{z}_{2}\right)
$$

and maintains an error $\epsilon(\boldsymbol{z})$ depending on $T$

$$
\epsilon(\boldsymbol{z})=1-\sum_{i=1}^{T} \lambda_{i} \boldsymbol{\phi}_{i}^{2}(\boldsymbol{z})
$$

With $\operatorname{Cov}\left(\boldsymbol{z}_{1}, \boldsymbol{z}_{2}\right)=\sigma_{X}\left(\boldsymbol{z}_{1}\right) \sigma_{X}\left(\boldsymbol{z}_{2}\right) \Gamma\left(\boldsymbol{z}_{1}, \boldsymbol{z}_{2}\right)$, Equation (6) is the normalised equivalent to the often used error variance $\epsilon_{\sigma^{2}}(\boldsymbol{z})$, defined e.g. by [2]. Furthermore, the mean error over the whole domain, equivalent to the mean error variance $\bar{\epsilon}_{\sigma^{2}}$, can be estimated by

$$
\bar{\epsilon}=1-\sum_{i=1}^{T} \lambda_{i} \int_{\mathcal{D}} \boldsymbol{\phi}_{i}^{2}(\boldsymbol{z}) \mathrm{d} \boldsymbol{z} .
$$


In case of an analytical solution, the eigenfunctions are orthonormal, $\boldsymbol{\phi}_{i}(\boldsymbol{z}) \boldsymbol{\phi}_{j}(\boldsymbol{z})=\delta_{i j}$, and Equation (7) simplifies to [2]

$$
\bar{\epsilon}=1-\frac{1}{|\mathcal{D}|} \sum_{i=1}^{T} \lambda_{i}
$$

Random fields are classically categorised with aleatory uncertainties, meaning that they describe the intrinsic randomness of a phenomena itself, which cannot be further reduced. However, the describing parameters may contain epistemic uncertainty caused by a lack of knowledge or data. For this reason, the concept of imprecise random fields is introduced in the following subsection.

\subsection{Probability box approach}

If one or several parameters cannot be determined precisely, the classically aleatory random field includes also epistemic uncertainties, e.g. by interval or fuzzy valued parameters [10]. To avoid further assumptions on the fuzziness, this work considers epistemic parameters to be interval valued. This leads to the concept of an imprecise random field [6],

$$
[X](\boldsymbol{z}, \omega)=\mu_{X}^{I}(\boldsymbol{z})+\sigma_{X}^{I} \sum_{i=1}^{\infty} \sqrt{\lambda_{i}^{I}} \boldsymbol{\phi}_{i}^{I}(\boldsymbol{z}) \xi_{i}(\omega),
$$

where the index $I$ denotes the interval valued random field parameters. The interval valued eigenvalues and -functions originate from an interval valued correlation length $L^{I}$.

In this work imprecise random fields are propagated through an FE problem using the probability box (p-box) approach [1]. By this, the quantity of interest $Y$ can be described by a left and right bound $\left[\bar{F}_{Y}, \underline{F}_{Y}\right]$ of the cumulative distribution function (CDF). If further information is available, e.g. the interval ranges $\mu_{Y}^{I}$ and $\sigma_{Y}^{I}$ of the mean value and the standard deviation or the probability family $\mathcal{F}$, these can be added and the p-box is described by the quintuple $\left(\bar{F}_{Y}, \underline{F}_{Y}, \mu_{Y}^{I}, \sigma_{Y}^{I}, \mathcal{F}\right)$.

The eigenvalues $\lambda_{i}$ are not monotonically dependent on the correlation length $L$. For this reason, a pure vertex analysis does not necessarily guarantee to gain the outer bounds of the p-box in case an imprecise random field contains an interval valued correlation length. A straightforward possibility is to discretise $L^{I}$, to perform a stochastic analysis with each $L_{i} \in L^{I}$ and to determine the p-box bounds by the minimum and maximum of all results [3]. Alternatively, if the used model is monotonic, the relevant intermediate correlation lengths $L_{i}^{*} \in L^{I}$ of the imprecise random field input can be determined by optimisation a priori [6]. This may reduce the computational effort of propagating multiple random fields, especially when several imprecise random fields are involved.

Note that when the constant standard deviation is considered to be interval valued (as well), the choice to decompose the autocorrelation function instead of the autocovariance function as it is usually done in literature - becomes beneficial in terms of the computational cost. As $\Gamma_{X}\left(\boldsymbol{z}_{1}, \boldsymbol{z}_{2}\right)$ is independent of $\sigma_{X}$, Equation (2) needs to be solved only once (per $L_{i}$ ) and not for each $\sigma_{X, i} \in \sigma_{X}^{I}$ (in combination with each $L_{i}$ ). 


\section{INVESTIGATION ON CRUCIAL RANDOM FIELD PARAMETERS}

Within this contribution one-dimensional (1D) random fields depending on the spatial parameter $z \in \mathcal{D}$ are investigated. Furthermore, an exponential autocorrelation function $\Gamma_{X}\left(z_{1}, z_{2}\right)$ is considered,

$$
\Gamma_{X}\left(z_{1}, z_{2}\right)=\exp \left\{-\frac{\left|z_{1}-z_{2}\right|}{L}\right\},
$$

which describes the decay of the autocorrelation in terms of the correlation length $L$. Note that the stochastic dimension $N$ of the random field depends on the truncation order $T$, see Equation (4). Due to the non-differentiability of Equation (10) at $z_{1}=z_{2}$, the corresponding random field can become very high-dimensional when $L$ is small compared to the domain length $l$. However, the availability of an analytical solution, described e.g. in [11], justifies the effort for the purpose of this paper.

The parameters which describe a random field are investigated in this section. Equation (1) can be interpreted as an expanded standard normal distributed random field $\left(\mu_{S}=0, \sigma_{S}=1\right)$ that is scaled by $\sigma_{X}$ and shifted towards $\mu_{X}(z)$. The mean value and the standard deviation therefore do not influence the expansion itself. Beside the chosen autocorrelation function, the main effect on the random field is caused by the correlation length. Therefore, in this section a $1 \mathrm{D}$ standard normal distributed random field $S(z, \omega)$ defined on $\mathcal{D}=[0,1]$ is investigated for the correlation length values $L=[0.01,0.1,1.0,10.0]$ in Subsection 3.2. The results are compared to the limits $L \rightarrow 0$, which defines white noise, and $L \rightarrow \infty$, which is equal to a constant standard normal distributed random variable $S(\omega)$. However, the impact of the truncation order $T$ on the expansion error needs to be studied before.

\subsection{Influence of the truncation order}

When different correlation lengths $L_{i} \in L^{I}$ are considered for an imprecise random field, special care must be taken regarding the truncation order $T$. To ensure that the p-box bounds are not affected by different errors within the input variance $\sigma^{2}\{\hat{X}(z, \omega)\}=\sigma_{X}^{2} \hat{\Gamma}_{X}\left(z_{1}, z_{2}\right)$, the truncation needs to be chosen individually for each $L_{i}$, e.g. in terms of an equal mean error $\bar{\epsilon}_{i}$. As it can be seen in Figure 1a, the convergence of the latter and consequently the stochastic dimension $N=T$ is highly dependent on the correlation length ratio. For large $L / l$ the corresponding $\bar{\epsilon}$ is already small for very few truncation terms. It is therefore practical to use the upper bound of $L^{I}$ to decide on $\bar{\epsilon}$. However, by this approach one can be forced to accept extremely high dimensions for the lower bound of $L^{I}$ when the range of the interval valued correlation length is large.

Table 1: Truncation orders $T$ resulting to the mean errors $\bar{\epsilon} \approx 3.2 \%, \bar{\epsilon} \approx 1.3 \%$ and $\bar{\epsilon} \approx 0.8 \%$ regarding an exponential autocorrelation function with different correlation length ratios $L / l$.

\begin{tabular}{lllllll}
\hline & \multicolumn{2}{c}{$\bar{\epsilon} \approx 3.2 \%$} & \multicolumn{2}{c}{$\bar{\epsilon} \approx 1.3 \%$} & \multicolumn{2}{c}{$\bar{\epsilon} \approx 0.8 \%$} \\
$L / l[-]$ & $T[-]$ & $\bar{\epsilon}[\%]$ & $T[-]$ & $\bar{\epsilon}[\%]$ & $T[-]$ & $\bar{\epsilon}[\%]$ \\
\hline 10.0 & 1 & 3.2441 & 2 & 1.2977 & 3 & 0.7963 \\
1.0 & 7 & 3.0997 & 16 & 1.3064 & 26 & 0.7948 \\
0.1 & 63 & 3.2382 & 156 & 1.3029 & 254 & 0.7993 \\
0.01 & 625 & 3.2420 & 1551 & 1.3068 & 2534 & 0.7998 \\
\hline
\end{tabular}


In Table 1 , for three possibly aimed errors $\bar{\epsilon} \approx 3.2 \%, \bar{\epsilon} \approx 1.3 \%$ and $\bar{\epsilon} \approx 0.8 \%$ (guided by the lowest possible truncation terms $T=1, T=2$ and $T=2$ of the maximum $L / l$ ) the closest possible error $\bar{\epsilon}_{i}$ and the corresponding value $T_{i}$ are listed for the different considered $L_{i} / l$. As can be seen for $\bar{\epsilon} \approx 3.2 \%$, not all $L_{i} / l$ can match this error well. The next possible error referred to $L / l=10$ is $\bar{\epsilon} \approx 1.3 \%$. Here the errors of the different $L_{i} / l$ are better comparable already but $T$ has more than doubled. Furthermore, if further relatively large correlation length ratios, e.g. $L / l=5$, were included, the corresponding error could again not match perfectly. For this reason, the truncation has to be investigated for each problem considered with imprecise random fields and the comparable error needs to be chosen individually depending on the involved values $L_{i}$ to be propagated. Regarding the local error $\epsilon(z)$ depicted in Figure 1b, another difficulty becomes clear in terms of different correlation lengths to be considered. Although all $L_{i} / l$ fulfil the same mean error $\epsilon(z) \approx 1.3 \%$, the local error still varies significantly when $T$ is small. Depending on the quantity of interest, this can lead to localisation effects [4].

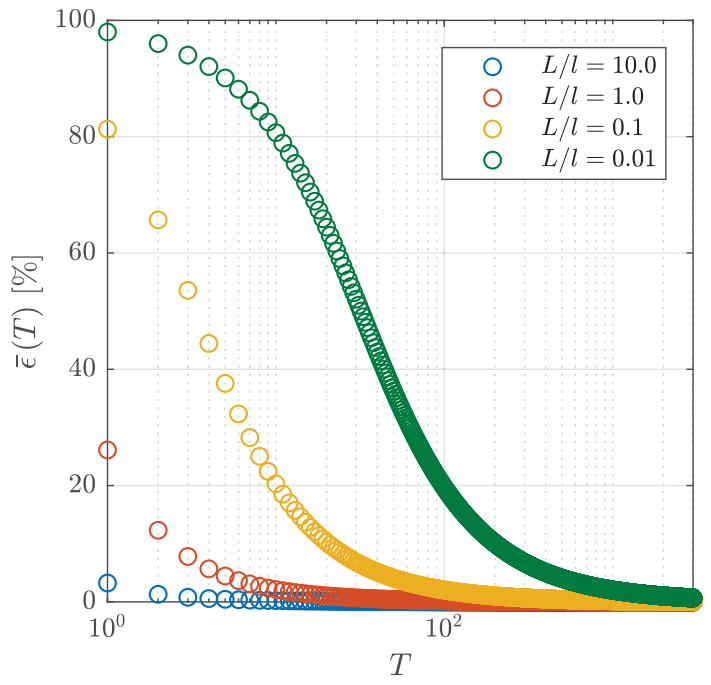

(a) convergence of $\bar{\epsilon}$ with increasing $T$

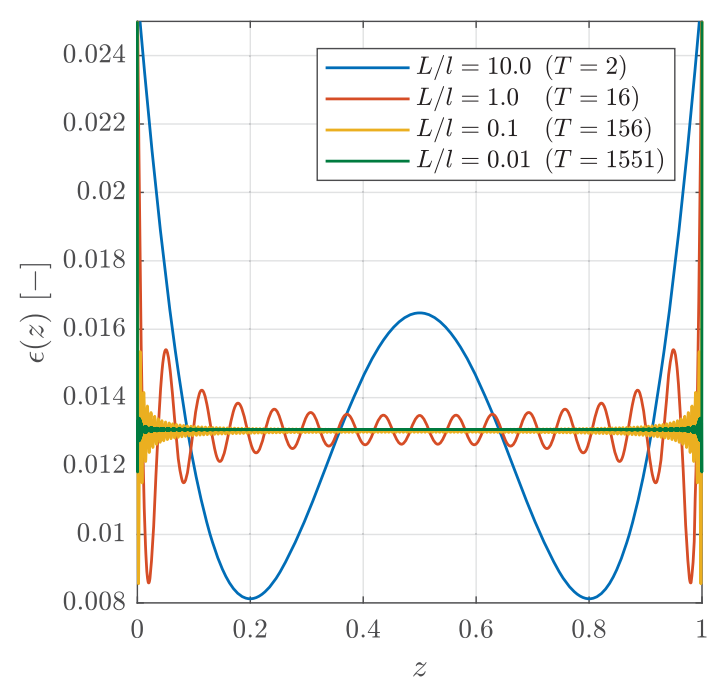

(b) local variation of $\epsilon(z)$ for a fixed $\bar{\epsilon} \approx 1.3 \%$

Figure 1: Influence of different correlation length ratios $L / l$ and truncation orders $T$ on the mean error $\bar{\epsilon}$ and the local error $\epsilon(z)$ of a 1D random field using an exponential autocorrelation function.

\subsection{Influence of the correlation length}

As already mentioned, the correlation length $L$ is a parameter indicating how fast or slow the autocorrelation between the random field values $X\left(z_{1}, \omega\right)$ and $X\left(z_{2}, \omega\right)$ decays with the distance $\left|z_{1}-z_{2}\right|$. When $L / l \rightarrow 0$, the values of the field are completely uncorrelated which is called white noise. On the other hand the random field converges towards a random variable for $L / l \rightarrow \infty$. In this subsection, the influence of $L_{i} / l$ on a standard normal distributed random field $S(z, \omega)$ is studied and the convergence of the random field properties towards these two limits are investigated. The corresponding truncation orders $T_{i}$ are chosen according to a mean error $\bar{\epsilon}_{i} \approx 0.8 \%$, compare Table 1 .

In Figure 2 the effect of the different considered correlation length ratios as well as the limits of $L / l$ is visualised. On the left side, the autocorrelation function $\Gamma\left(z_{1}, z_{2}\right)$ is depicted in its closed form. It can be easily seen that the non-differentiability at $z_{1}=z_{2}$ becomes more and more crucial for decreasing $L / l$. On the right, three standard normal distributed random field 

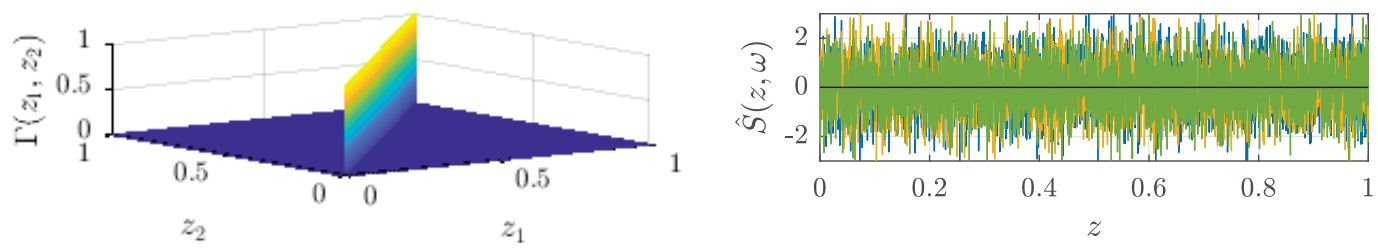

(a) $L / l \rightarrow 0$ (white noise)
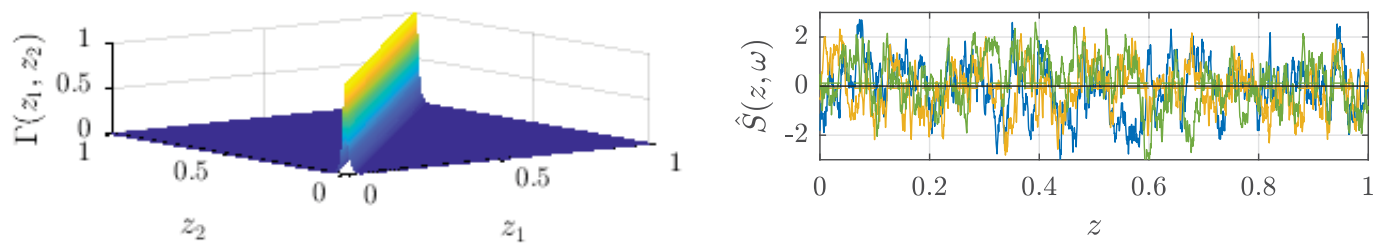

(b) $L / l=0.01$
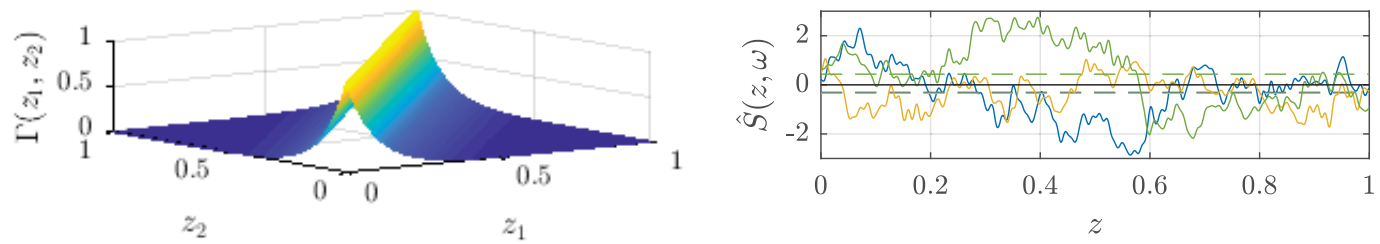

(c) $L / l=0.1$
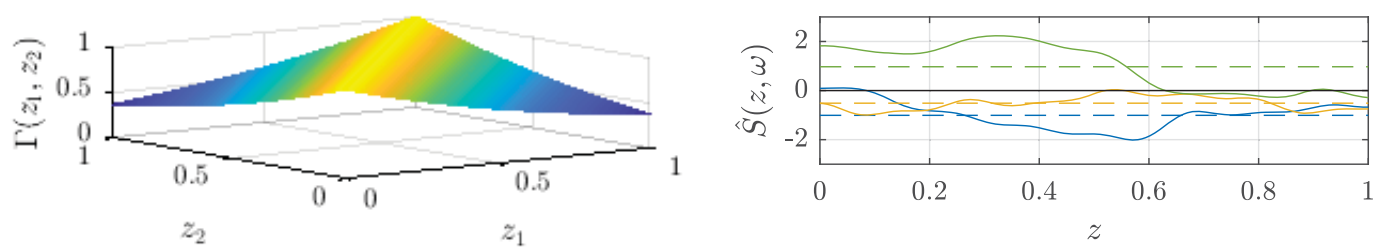

(d) $L / l=1.0$
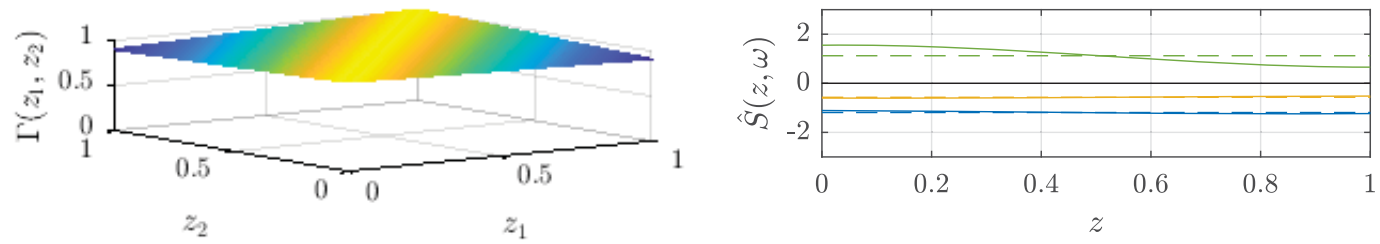

(e) $L / l=10.0$
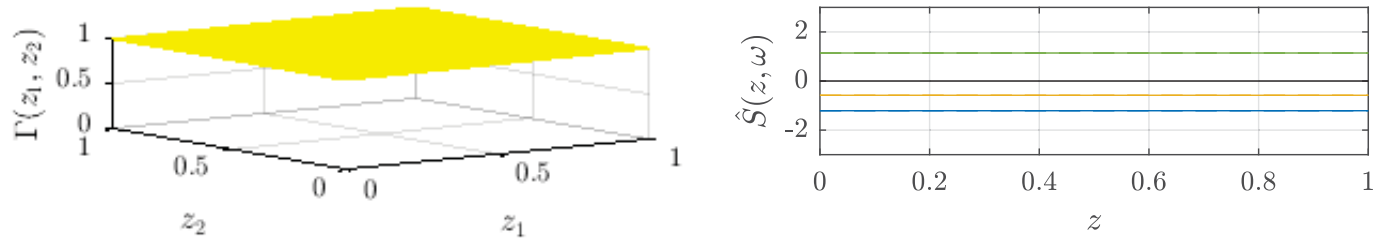

(f) $L / l \rightarrow \infty$ (random variable)

Figure 2: Influence of the correlation length ratio $L / l$ considering a 1D standard normal distributed random field. Left: closed form of the exponential autocorrelation function $\Gamma\left(z_{1}, z_{2}\right)$, right: three random field realisations $\hat{S}_{j}=\hat{S}\left(z, \omega_{j}\right)$ (solid lines) and their corresponding mean values $\hat{\mu}_{S, j}=\mu\left\{\hat{S}_{j}\right\}$ (dashed lines). 
realisations $\hat{S}_{j}=\hat{S}\left(z, \omega_{j}\right)$ are depicted for the different ratios $L / l$. Furthermore, the individual mean value $\hat{\mu}_{S, j}=\mu\left\{\hat{S}_{j}\right\}$ corresponding to the realisation $j$ is given in a dashed line of the same colour. It can be seen that the variation of the random field increases with a decreasing $L / l$. However, the higher the variation is, the more likely $\hat{\mu}_{S, j}$ falls close to the input mean value $\mu_{S}=0$ of the random field. Therefore, the convergence behaviour of $\hat{\mu}_{S, j}$ is investigated further in terms of the number $n_{\mathrm{MC}}$ of random field realisations.

The variation of the mean value $\hat{\mu}_{S, j}$ of an individual random field $\hat{S}_{j}=\hat{S}\left(z, \omega_{j}\right)$ seems to depend on the correlation length ratio $L / l$. In the following the mean value $\mu\left\{\hat{\mu}_{S, j}\right\}$ and standard deviation $\sigma\left\{\hat{\mu}_{S, j}\right\}$ of the individual random field mean values $\hat{\mu}_{S, j}, j=1, \ldots, n_{\mathrm{MC}}$, are discussed in terms of an increasing sample size $n_{\mathrm{MC}}$. As can be seen in Figure 3a, considering a sufficiently large sample size the mean value $\mu\left\{\hat{\mu}_{S, j}\right\}$ of all random field mean values converges towards the input mean value $\mu_{S}=0$ that has been used in Equation (4) to create the realisations. On the contrary, the standard deviation $\sigma\left\{\hat{\mu}_{S, j}\right\}$ of all random field mean values is not generally converging towards the input standard deviation $\sigma_{S}=1$ but towards individual values $\sigma \in[0,1]$. Furthermore, $L / l \rightarrow 0$ describes the lower bound with $\sigma\left\{\hat{\mu}_{S, j}\right\}$ converging to zero, $L / l \rightarrow \infty$ the upper bound with $\sigma\left\{\hat{\mu}_{S, j}\right\}$ converging to one.

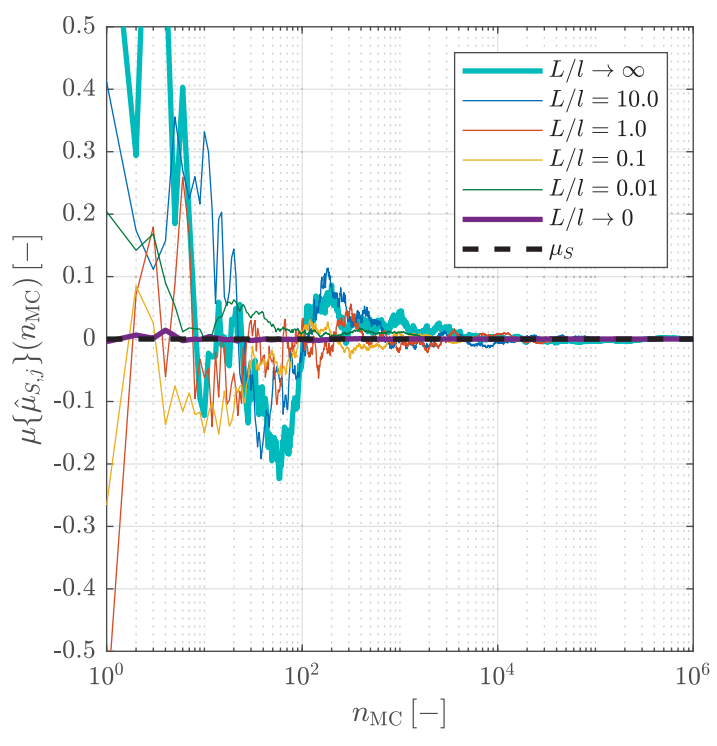

(a) $\mu\left\{\hat{\mu}_{S, j}\right\}$ converging to $\mu_{S}$

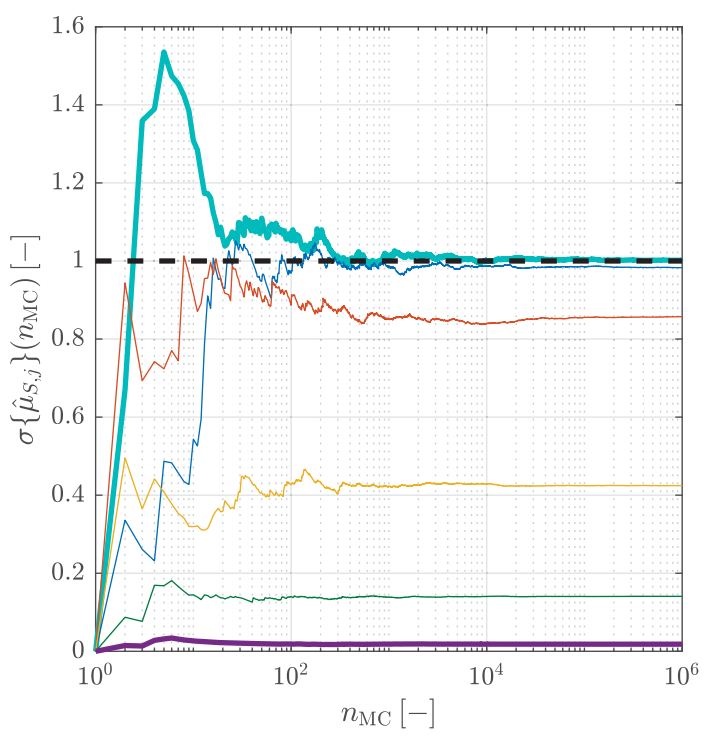

(b) $\sigma\left\{\hat{\mu}_{S, j}\right\}$ converging to a value $\sigma \in\left[0, \sigma_{S}\right]$

Figure 3: Convergence of the mean value $\mu\left\{\hat{\mu}_{S, j}\right\}$ and the standard deviation $\sigma\left\{\hat{\mu}_{S, j}\right\}$ of the individual mean values $\hat{\mu}_{S, j}=\mu\left\{\hat{S}_{j}\right\}$ of $n_{\mathrm{MC}}$ standard normal distributed random field realisations $\hat{S}_{j}=\hat{S}\left(z, \omega_{j}\right)$.

In this case, a standard normal distributed random field $\hat{S}(x, \omega)$ has been used. It has been found that $\mu\left\{\hat{\mu}_{S, j}\right\} \rightarrow \mu_{S}=0$ and $\sigma\left\{\hat{\mu}_{S, j}\right\} \rightarrow \sigma \in\left[0, \sigma_{S}=1\right]$. However, understanding an arbitrary random field $X(z, \omega)$ as a standard normal distributed random field $S(z, \omega)$ that has been scaled by $\sigma_{X}$ and shifted towards $\mu_{X}(z)$, one can conclude in general that for $j=$ $1, \ldots, n_{\mathrm{MC}}$ and $n_{\mathrm{MC}}$ sufficiently large

$$
\mu\left\{\hat{\mu}_{X, j}\right\} \rightarrow \mu_{X} \quad \text { independent of } L / l,
$$


and $\sigma\left\{\hat{\mu}_{X, j}\right\}$ is bounded by the limits of $L / l$,

$$
\sigma\left\{\hat{\mu}_{X, j}\right\} \rightarrow \begin{cases}0 & \text { for } L / l \rightarrow 0 \\ \sigma_{X} & \text { for } L / l \rightarrow \infty\end{cases}
$$

For this reason it can be worth the effort to once determine $\sigma\left\{\hat{\mu}_{S, j}\right\}$ of a standard normal distributed random variable as a function of the correlation length ratio $L / l$. Having this standardised result for a given autocorrelation function one can estimate $\sigma\left\{\hat{\mu}_{X, j}\right\}$ of any random field $X(z, \omega)$ by

$$
\sigma\left\{\hat{\mu}_{X, j}\right\}(L / l)=\sigma_{X} \cdot \sigma\left\{\hat{\mu}_{S, j}\right\}(L / l),
$$

where $\sigma_{X}$ is the input standard deviation that is used to create random field realisations in Equation (4). Then, if the random field propagates linearly through the applied model, the mean value and standard deviation of a quantity of interest can be estimated immediately for any further $L_{i} / l$ as soon as two correlation length values have been propagated.

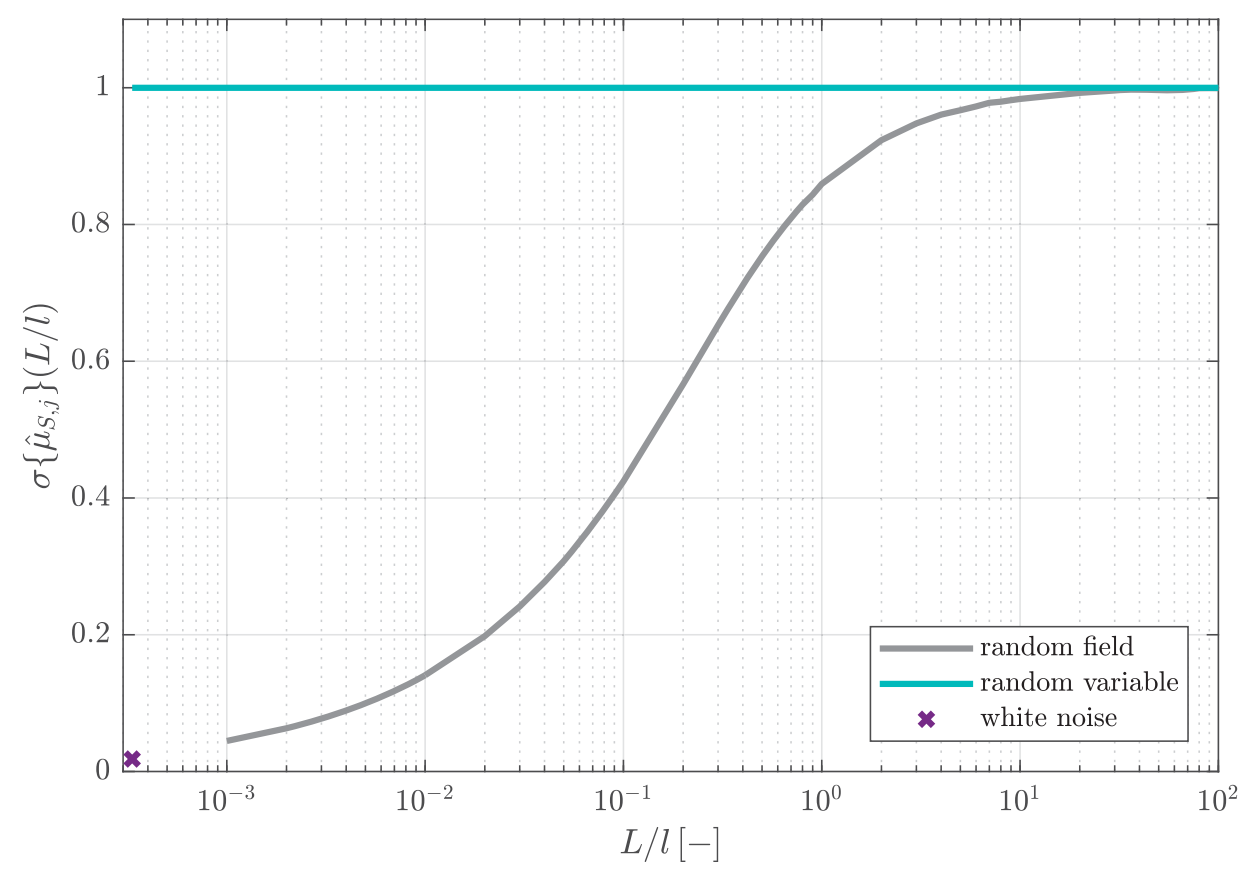

Figure 4: Standard deviation $\sigma\left\{\hat{\mu}_{S, j}\right\}$ of the individual mean values $\hat{\mu}_{S, j}=\mu\left\{\hat{S}_{j}\right\}$ of $n_{\mathrm{MC}}=10^{6}$ standard normal distributed random field realisations $\hat{S}_{j}(z, \omega)$ with respect to the correlation length ration $L / l$.

In Figure 4 the standard deviation $\sigma\left\{\hat{\mu}_{S, j}\right\}$ resulting from $n_{\mathrm{MC}}=10^{6}$ individual random field mean values $\hat{\mu}_{S, j}=\mu\left\{\hat{S}_{j}\right\}$ is depicted as a function of $L / l$. Note that the results of a white noise property depend on the discretisation of the domain, here $n_{\mathrm{el}}=3000$, and therefore $\sigma\left\{\hat{\mu}_{S, j}\right\}$ is not exactly zero. However, it can be expected that for $n_{\mathrm{el}} \rightarrow \infty$ it is $\sigma\left\{\hat{\mu}_{S, j}\right\} \rightarrow 0$. It can be estimated that for correlation lengths $L$ which are larger than ten times the domain length $l$, the resulting random field starts to converge towards a random variable and the effort of discretising the random field by KL expansion might not be justified. On the lower bound, $L / l$ is rather restricted by the feasibility in terms of the stochastic dimension than by converging towards white noise. 


\section{LINEAR BEAM STUDY}

Considering a linear model $Y=\mathcal{M}(X)$ to propagate (imprecise) random fields $X(z, \omega)$, it can be assumed that the mean value $\mu\{Y\}$ and the standard deviation $\sigma\{Y\}$ depend linearly on $\mu\left\{\hat{\mu}_{X, j}\right\}$ and $\sigma\left\{\hat{\mu}_{X, j}\right\}$. Instead of simulating several $L_{i} \in L^{I}$ to determine the p-box of $Y$, the computational cost could therefore be reduced significantly by just propagating the limits of $L$, meaning white noise and a random variable, through the model to gain the p-box of "absolutely no idea". Any further needed $L_{i} / l$ could then be simply gained by linear interpolation. The standard deviation $\sigma\left\{\hat{\mu}_{X, j}\right\}$ corresponding to a specific random field $X(z, \omega)$ with any standard deviation $\sigma_{X}$ defined on an arbitrary $\mathcal{D}$ can be gained by sampling, which is much cheaper when the random field does not need to be propagated. Alternatively, it even can be estimated by the standardised results depicted in Figure 4 by reading $\sigma\left\{\hat{\mu}_{S, j}\right\}$ corresponding to $L / l$ from the graph and multiplying this value with $\sigma_{X}$ as given in Equation (13).

This assumption is further studied within this section. For this purpose, the beam problem depicted in Figure 5 is considered assuming a linear elastic material. To gain a good representation of white noise, the beam is discretised by $n_{\mathrm{el}}=30001 \mathrm{D}$ beam elements. The investigated random fields are assumed as a function along the beam length $l=1 \mathrm{~m}$ and to be constant within the cross section $A=0.01 \mathrm{~m}^{2}$ (1D random field). The maximum deflection $w_{\max }=w(z=0.5 \mathrm{~m})$ in the middle of the both-sided supported beam is the quantity of interest. For a deterministic simulation using a Young's modulus $E=210 \cdot 10^{9} \frac{\mathrm{N}}{\mathrm{m}^{2}}$ and a constant line load $q_{0}=5000 \frac{\mathrm{N}}{\mathrm{m}}$, the quantity of interest results in $w_{\max }=3.72 \cdot 10^{-5} \mathrm{~m}$.

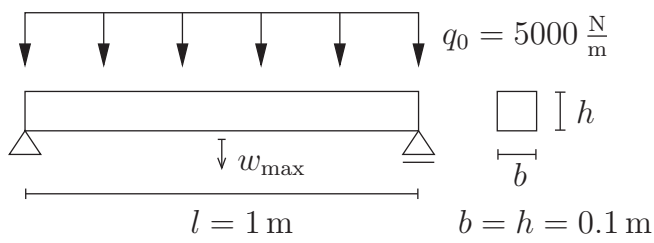

Figure 5: Linear-elastic beam with Young's modulus $E=210 \cdot 10^{9} \frac{\mathrm{N}}{\mathrm{m}^{2}}$ under constant line load, deterministic maximal deflection: $w_{\max }=3.72 \cdot 10^{-5} \mathrm{~m}$.

In the following subsections, both, the line load and the Young's modulus are considered as imprecise random fields in first two studies independently, and in a third study combined.

\subsection{Investigation on different imprecise random field input parameters}

In the following, two studies on the correlation length ratio $L / l$ are performed, each with one parameter considered as imprecise random field input with the parameters given in Table 2. For both studies, the discretised correlation length intervals $L^{(1)} / l=[0.01: 0.01: 0.1], L^{(2)} / l=$ $[0.1: 0.1: 1.0]$ and $L^{(3)} / l=[1.0: 1.0: 10.0]$ as well as $L / l \rightarrow 0$ (white noise, abbreviated by $\mathrm{WN}$ ) and $L / l \rightarrow \infty$ (random variable, abbreviated by RV) are investigated chosing $\bar{\epsilon}=0.8 \%$. A closer look is spend on the results of the values $L^{(*)} / l=[\mathrm{WN}, 0.01,0.1,1.0,10.0, \mathrm{RV}]$. Each $L_{i} / l$ simulation is performed using brute force $\mathrm{MC}$ with $n_{\mathrm{MC}}=10000$ samples.

In the first study, the line load $q$ is modelled as an imprecise random field with $\mu_{q}=q_{0}=$ $5000 \frac{\mathrm{N}}{\mathrm{m}}$ and $\sigma_{q}=0.1 \mu_{q}=500 \frac{\mathrm{N}}{\mathrm{m}}$. As $q$ is in the numerator of the beam deflection solution, it can be assumed that the propagation of $L_{i} / l$ through the FE model is linear. The Young's modulus $E$, considered with $\mu_{E}=E=210 \cdot 10^{9} \frac{\mathrm{N}}{\mathrm{m}^{2}}$ and $\sigma_{E}=0.05 \mu_{E}=10.5 \cdot 10^{9} \frac{\mathrm{N}}{\mathrm{m}^{2}}$ within the second study, can be found in the denominator of the beam deflection solution. Therefore, the results are expected to depend inversely on $E(z, \omega)$ and might not be Gaussian distributed anymore. 
Table 2: Random field parameters considered for two studies including each one random field input parameter.

\begin{tabular}{llll}
\hline & random field input & mean value & standard deviation \\
\hline study 1 & line load $q(z, \omega)$ & $\mu_{q}=5000 \frac{\mathrm{N}}{\mathrm{m}}$ & $\sigma_{q}=0.1 \mu_{q}=500 \frac{\mathrm{N}}{\mathrm{m}}$ \\
study 2 & Young's modulus $E(z, \omega)$ & $\mu_{E}=210 \cdot 10^{9} \frac{\mathrm{N}}{\mathrm{m}^{2}}$ & $\sigma_{E}=0.05 \mu_{E}=10.5 \cdot 10^{9} \frac{\mathrm{N}}{\mathrm{m}^{2}}$ \\
\hline
\end{tabular}

The CDFs resulting from each $L_{i} / l$ are depicted in Figure 6 for both studies. The axis describing $w_{\max }$ is scaled uniquely such that both p-boxes can be directly compared qualitatively. The deterministic result is given as a vertical dash-dot line, the results of $L^{(*)} / l$ in different colours and these of all other $L_{i} / l$ as grey dotted lines. Both results appear to be Gaussian distributed, which corroborates the assumption of a linear dependency between input and output.

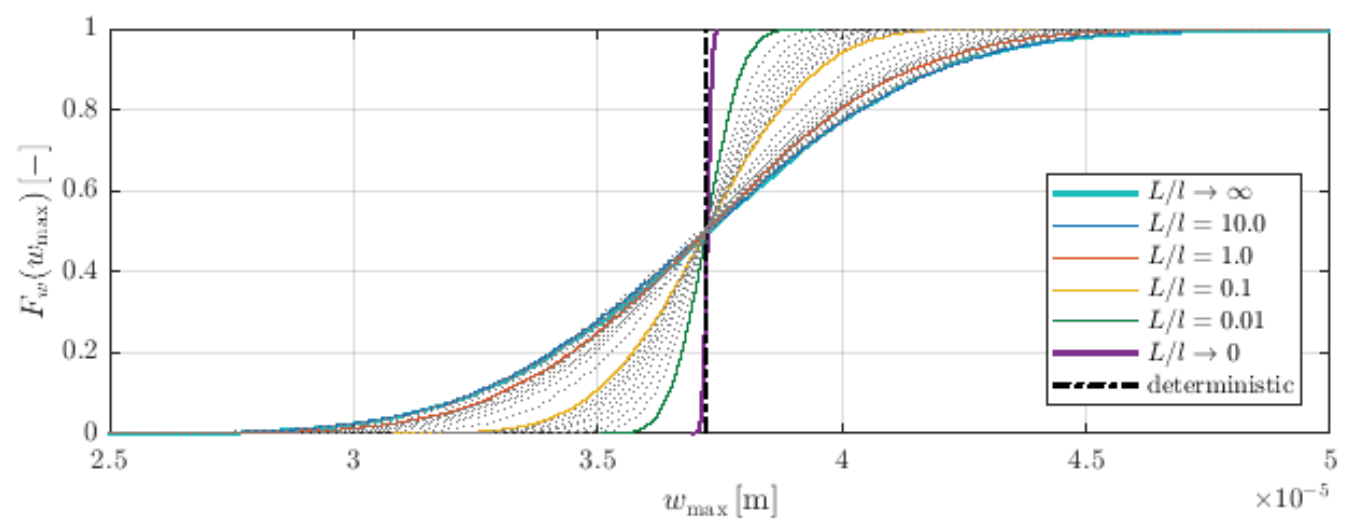

(a) study 1: line load as input random field

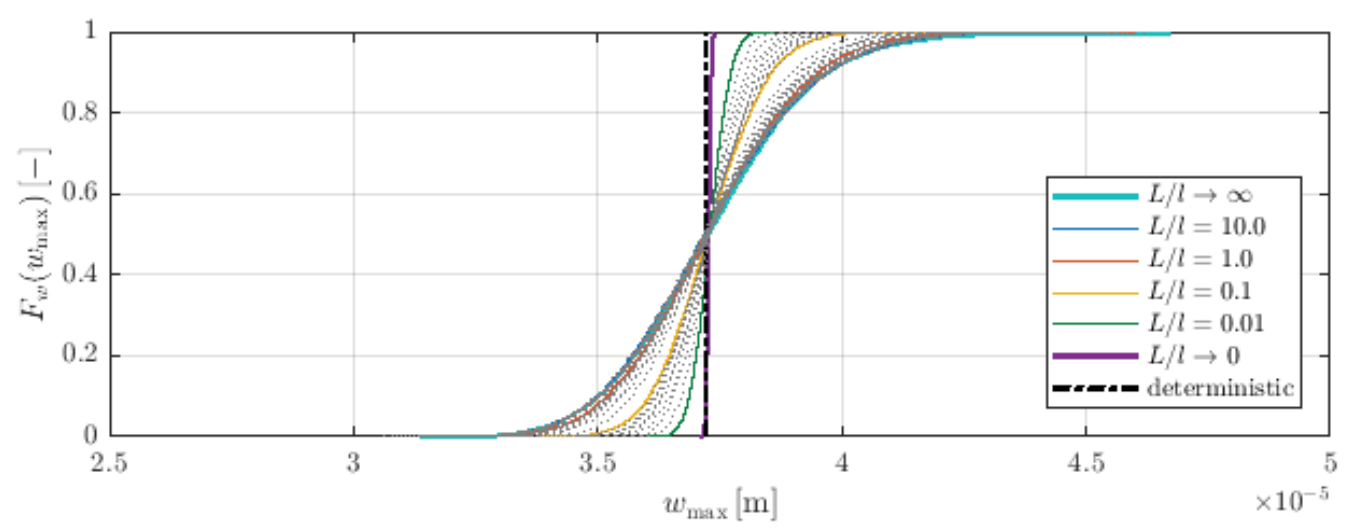

(b) study 2: Young's modulus as input random field

Figure 6: CDFs for different correlation length ratios $L / l$ bounded by white noise $L / l \rightarrow 0$ and a random variable $L / l \rightarrow \infty$ considering one random field input parameter.

In the first study, the input standard deviation is assumed to be ten percent of the input mean value, $\sigma_{q}=0.1 \mu_{q}$, while it is only five percent, $\sigma_{E}=0.05 \mu_{E}$, in the second study. For this reason, the resulting p-box is much wider in Figure 6a than in Figure 6b. The CDF gained by a random variable spans the widest range of $w_{\max }$. With decreasing $L / l$ the CDFs become steeper. The CDF of the white noise is very close to the deterministic value. It can be assumed that it will converge towards a vertical line for $n_{\mathrm{el}} \rightarrow \infty$. By this, one can estimate an "absolutely no idea 
p-box" by even just one stochastic simulation, the one assuming just a random variable. The white noise result defining the second part of the p-box bound can be considered as a vertical line $\mu\left\{w_{\max }^{\mathrm{RV}}\right\}$. In addition to the qualitative impression gained by Figure 6 , the mean value and standard deviation of both, input random fields and the quantity of interest according to each $L^{(*)} / l$ can be compared quantitatively in Table 3 .

Table 3: Mean value $\mu\left\{\hat{\mu}_{X, j}\right\}$ and standard deviation $\sigma\left\{\hat{\mu}_{X, j}\right\}$ of the considered input random fields as well as mean value $\mu\left\{w_{\max }\right\}$ and standard deviation $\sigma\left\{w_{\max }\right\}$ of the maximum beam deflection $w_{\max }$ resulting from a propagation through a linear FE model for different $L / l$.

(a) study 1: line load as input random field

\begin{tabular}{lllll}
\hline & \multicolumn{2}{c}{ input realisations } & \multicolumn{2}{c}{ quantity interest } \\
$L / l[-]$ & $\mu\left\{\hat{\mu}_{q, j}\right\}\left[\frac{\mathrm{N}}{\mathrm{m}}\right]$ & $\sigma\left\{\hat{\mu}_{q, j}\right\}\left[\frac{\mathrm{N}}{\mathrm{m}}\right]$ & $\mu\left\{w_{\max }\right\}[\mathrm{m}]$ & $\sigma\left\{w_{\max }\right\}[\mathrm{m}]$ \\
\hline $\mathrm{WN}$ & $5.0001 \mathrm{e}+03$ & $9.1424 \mathrm{e}+00$ & $3.7223 \mathrm{e}-05$ & $7.5621 \mathrm{e}-08$ \\
0.01 & $4.9986 \mathrm{e}+03$ & $7.0213 \mathrm{e}+01$ & $3.7213 \mathrm{e}-05$ & $5.8664 \mathrm{e}-07$ \\
0.1 & $4.9998 \mathrm{e}+03$ & $2.1207 \mathrm{e}+02$ & $3.7214 \mathrm{e}-05$ & $1.7917 \mathrm{e}-06$ \\
1.0 & $5.0002 \mathrm{e}+03$ & $4.2623 \mathrm{e}+02$ & $3.7221 \mathrm{e}-05$ & $3.2960 \mathrm{e}-06$ \\
10.0 & $5.0013 \mathrm{e}+03$ & $4.9965 \mathrm{e}+02$ & $3.7231 \mathrm{e}-05$ & $3.7368 \mathrm{e}-06$ \\
RV & $5.0039 \mathrm{e}+03$ & $5.0130 \mathrm{e}+02$ & $3.7251 \mathrm{e}-05$ & $3.7319 \mathrm{e}-06$ \\
\hline deterministic & $5 \mathrm{e}+03$ & - & $3.72 \mathrm{e}-05$ & - \\
\hline
\end{tabular}

(b) study 2: Young's modulus as input random field

\begin{tabular}{lllll}
\hline & \multicolumn{2}{c}{ input realisations } & \multicolumn{2}{c}{ quantity interest } \\
$L / l[-]$ & $\mu\left\{\hat{\mu}_{E, j}\right\}\left[\frac{\mathrm{N}}{\mathrm{m}^{2}}\right]$ & $\sigma\left\{\hat{\mu}_{E, j}\right\}\left[\frac{\mathrm{N}}{\mathrm{m}^{2}}\right]$ & $\mu\left\{w_{\max }\right\}[\mathrm{m}]$ & $\sigma\left\{w_{\max }\right\}[\mathrm{m}]$ \\
\hline $\mathrm{WN}$ & $2.1000 \mathrm{e}+11$ & $1.9224 \mathrm{e}+08$ & $3.7249 \mathrm{e}-05$ & $4.3169 \mathrm{e}-08$ \\
0.01 & $2.0998 \mathrm{e}+11$ & $1.4680 \mathrm{e}+09$ & $3.7299 \mathrm{e}-05$ & $3.3463 \mathrm{e}-07$ \\
0.1 & $2.1001 \mathrm{e}+11$ & $4.4876 \mathrm{e}+09$ & $3.7293 \mathrm{e}-05$ & $9.9669 \mathrm{e}-07$ \\
1.0 & $2.0994 \mathrm{e}+11$ & $8.9484 \mathrm{e}+09$ & $3.7310 \mathrm{e}-05$ & $1.6989 \mathrm{e}-06$ \\
10.0 & $2.0000 \mathrm{e}+11$ & $1.0341 \mathrm{e}+10$ & $3.7295 \mathrm{e}-05$ & $1.8649 \mathrm{e}-06$ \\
$\mathrm{RV}$ & $2.0990 \mathrm{e}+11$ & $1.0457 \mathrm{e}+10$ & $3.7313 \mathrm{e}-05$ & $1.8716 \mathrm{e}-06$ \\
\hline deterministic & $2.1 \mathrm{e}+11$ & - & $3.72 \mathrm{e}-05$ & - \\
\hline
\end{tabular}

As it was expected based on the behaviour of random fields investigated in Subsection 3.2, the mean values $\mu\left\{w_{\max }\right\}$ of the maximum beam deflection turn out to lay close to the deterministic result, independent of the correlation length. The standard deviation $\sigma\left\{\hat{\mu}_{X, j}\right\}$ of the input random fields have been determined by the generated samples. Alternatively, $\sigma\left\{\hat{\mu}_{X, j}\right\}$ can be determined by Equation (13). The values of both options are compared in Table 4 for both considered random field inputs, the line load $q(z, \omega)$ and the Young's modulus $E(z, \omega)$. Note that $\sigma\left\{\hat{\mu}_{S, j}\right\}$ has been determined by $n_{\mathrm{MC}}=10^{6}$ samples, while the $q(z, \omega)$ and $E(z, \omega)$ have been sampled only $n_{\mathrm{MC}}=10^{4}$ times each. Still, the results are comparable already. Furthermore, it can be seen that Equation (12) holds true for both input parameters $E$ and $q$.

Regarding the standard deviation $\sigma\left\{w_{\max }\right\}$ of the quantity of interest, it can be seen that the value is very small for white noise and increases with increasing $L / l$ for both studies in Ta- 
Table 4: Comparison of the standard deviation $\sigma\left\{\hat{\mu}_{X, j}\right\}$ of the input random fields determined by $n_{\mathrm{MC}}=10000$ samples with the result gained by factorising $\sigma\left\{\hat{\mu}_{S, j}\right\}$ of a standard normal distributed random field $\hat{S}(z, \omega)$ by $\sigma_{X}$.

\begin{tabular}{llllll}
\hline & & \multicolumn{2}{c}{ line load $q(z, \omega)\left[\frac{\mathrm{N}}{\mathrm{m}}\right]$} & \multicolumn{2}{c}{ Young's modulus $E(z, \omega)\left[\frac{\mathrm{N}}{\mathrm{m}^{2}}\right]$} \\
$L / l[-]$ & $\sigma\left\{\hat{\mu}_{S, j}\right\}[-]$ & $\sigma_{q} \cdot \sigma\left\{\hat{\mu}_{S, j}\right\}$ & $\sigma\left\{\hat{\mu}_{q, j}\right\}$ & $\sigma_{E} \cdot \sigma\left\{\hat{\mu}_{S, j}\right\}$ & $\sigma\left\{\hat{\mu}_{E, j}\right\}$ \\
\hline $\mathrm{WN}$ & 0.0183 & $9.1500 \mathrm{e}+00$ & $9.1424 \mathrm{e}+00$ & $1.9215 \mathrm{e}+08$ & $1.9224 \mathrm{e}+08$ \\
0.01 & 0.1408 & $7.0400 \mathrm{e}+01$ & $7.0213 \mathrm{e}+01$ & $1.4784 \mathrm{e}+09$ & $1.4680 \mathrm{e}+09$ \\
0.1 & 0.4243 & $2.1212 \mathrm{e}+02$ & $2.1207 \mathrm{e}+02$ & $4.4552 \mathrm{e}+09$ & $4.4876 \mathrm{e}+09$ \\
1.0 & 0.8593 & $4.2965 \mathrm{e}+02$ & $4.2623 \mathrm{e}+02$ & $9.0227 \mathrm{e}+09$ & $8.9484 \mathrm{e}+09$ \\
10.0 & 0.9836 & $4.9180 \mathrm{e}+02$ & $4.9965 \mathrm{e}+02$ & $1.0328 \mathrm{e}+10$ & $1.0341 \mathrm{e}+10$ \\
$\mathrm{RV}$ & 0.9999 & $4.9995 \mathrm{e}+02$ & $5.0130 \mathrm{e}+02$ & $1.0499 \mathrm{e}+10$ & $1.0457 \mathrm{e}+10$ \\
\hline
\end{tabular}

bles $3 \mathrm{a}$ and $3 \mathrm{~b}$. To investigate a possible linear dependence between input and output, $\sigma\left\{w_{\max }\right\}$ is plotted versus $\sigma\left\{\hat{\mu}_{X, j}\right\}$ in Figure 7. The axis denoting $\sigma\left\{w_{\max }\right\}$ is scaled equally for both studies. This way, the different percentages in the input standard deviations, $\sigma_{q}=0.1 \mu_{q}$ and $\sigma_{E}=0.05 \mu_{E}$ become visible again. The values $L^{(*)} / l$ are highlighted in red while all other $L_{i} / l$ pairs are depicted as grey crosses. The blue line represents the assumed linear dependence between white noise, for which it is $\sigma\left\{w_{\max }\right\} \rightarrow 0$ for $n_{\mathrm{el}} \rightarrow \infty$, and the random variable. It can be seen that the dependence between input and output standard deviation is not perfectly linear, as the results lay above the blue line. Assuming a linear dependence and interpolating any $L / l$ response from the CDF gained by a random variable would therefore underestimate the real standard deviation.

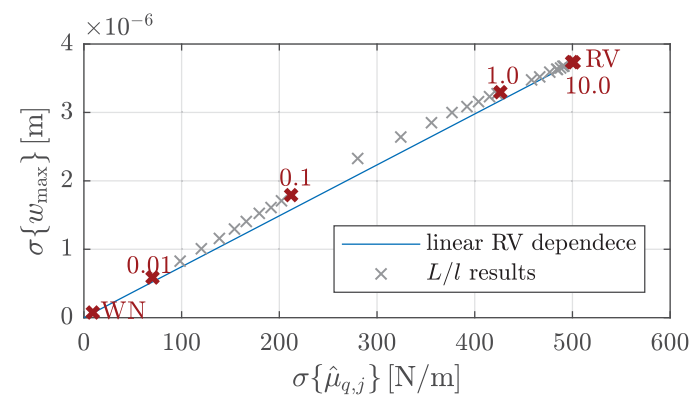

(a) study 1: line load as input random field

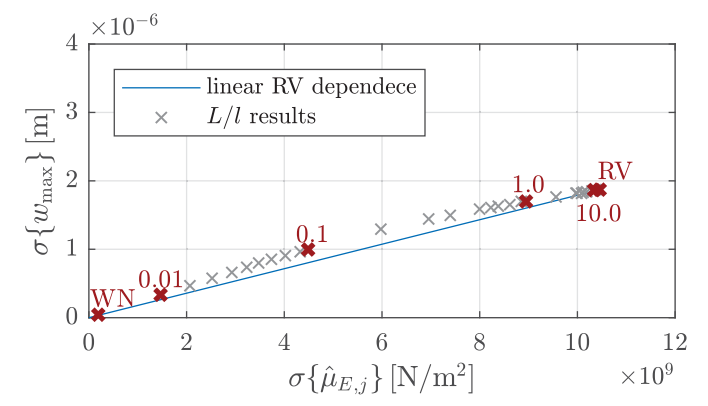

(b) study 2: Young's modulus as input random field

Figure 7: Dependence of the output standard deviation $\sigma\left\{w_{\max }\right\}$ on the input standard deviation $\sigma\left\{\hat{\mu}_{X, j}\right\}$ of the individual mean values $\hat{\mu}_{X, j}=\mu\left\{\hat{X}_{j}\right\}$ with $n_{\mathrm{MC}}=10000$ random field realisations $\hat{X}_{j}(z, \omega)$ when one input random field is considered.

The CDFs resulting from a linear interpolation of $L_{i} / l$ within the "absolutely no idea p-box" are compared to the ones gained by sampling and propagation in Figures 8 and 9 for both studies. The underestimated standard deviation is clearly visible by the dashed lines, which denote the interpolated CDFs, being slightly steeper than the corresponding CDF gained by sampling (solid line). However, with respect to the spectrum resulting from "having no idea at all" about the correlation length, the linear interpolation leads to a good estimate. Furthermore, if it is supposed to represent the lower bound of $L$, the estimate returns a slightly more conservative but save bound. 


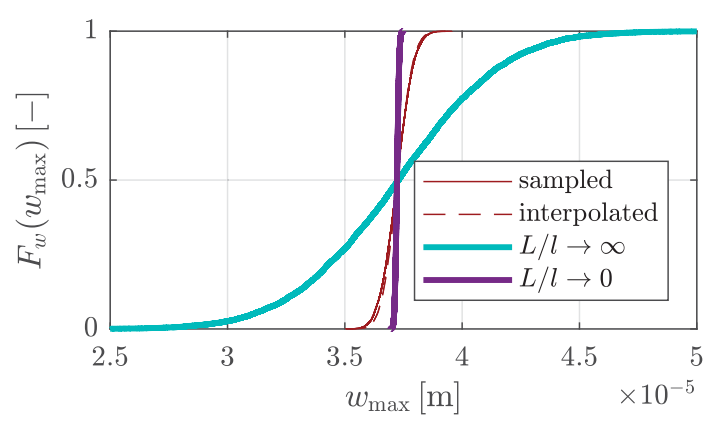

(a) $L_{q} / l=0.01$

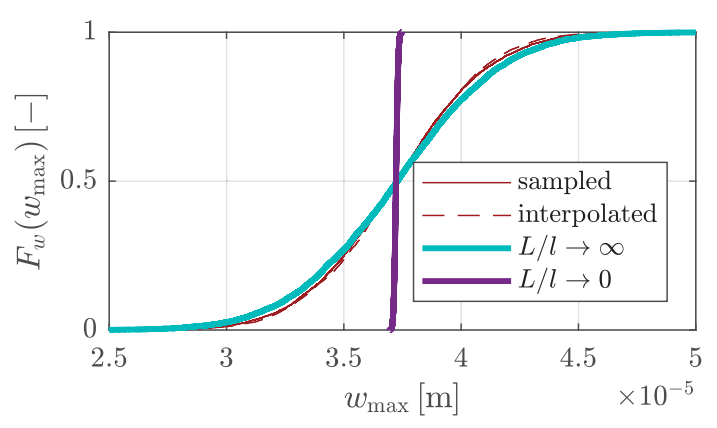

(c) $L_{q} / l=1.0$

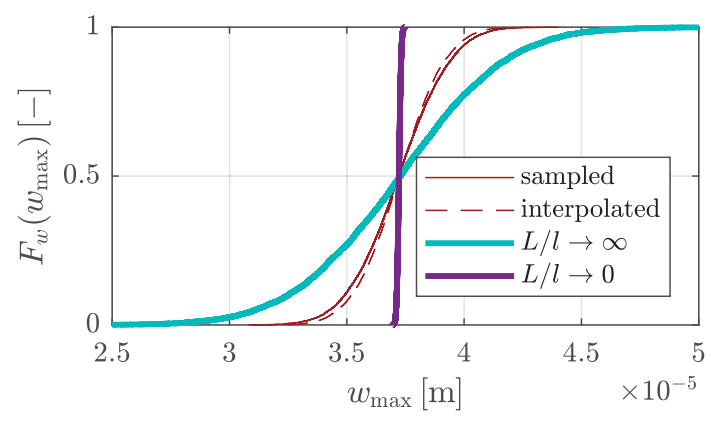

(b) $L_{q} / l=0.1$

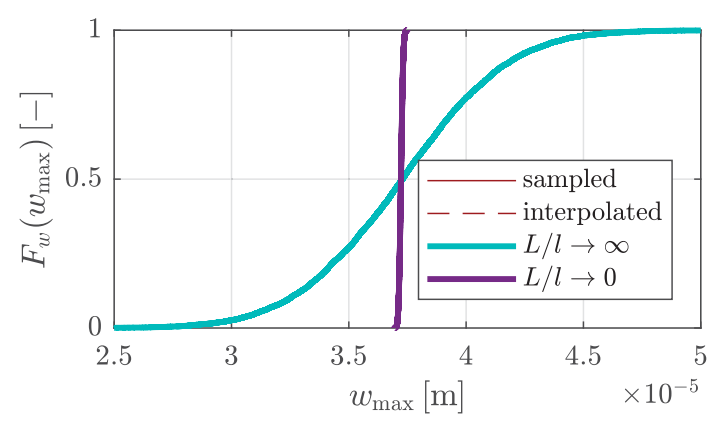

(d) $L_{q} / l=10.0$

Figure 8: Comparison of the CDFs gained by sampling and interpolation within the "absolutely no idea p-box" for different correlation lengths $L_{q} / l$ considering the line load as an imprecise random field input.

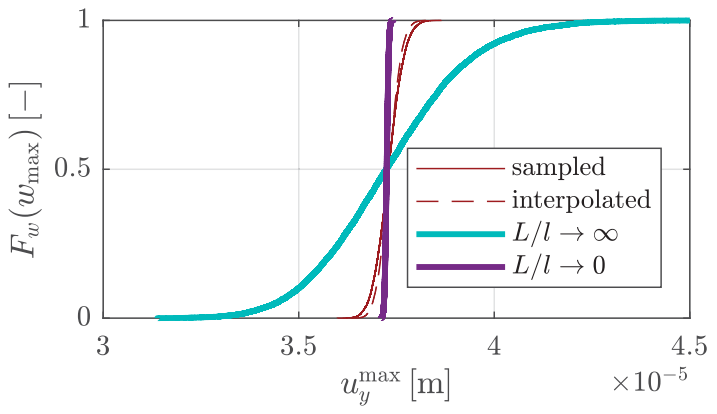

(a) $L_{E} / l=0.01$

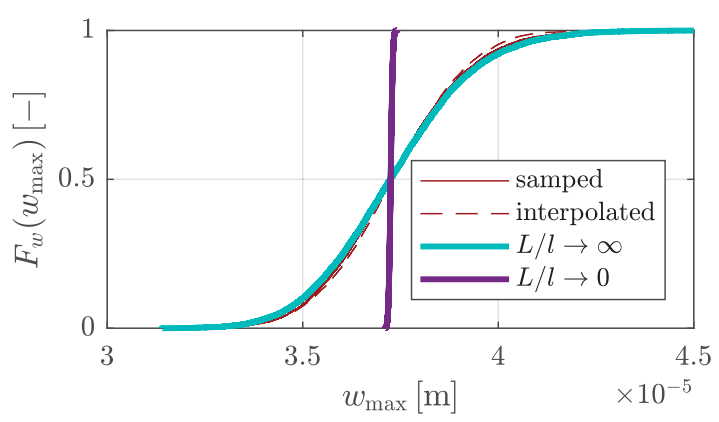

(c) $L_{E} / l=1.0$

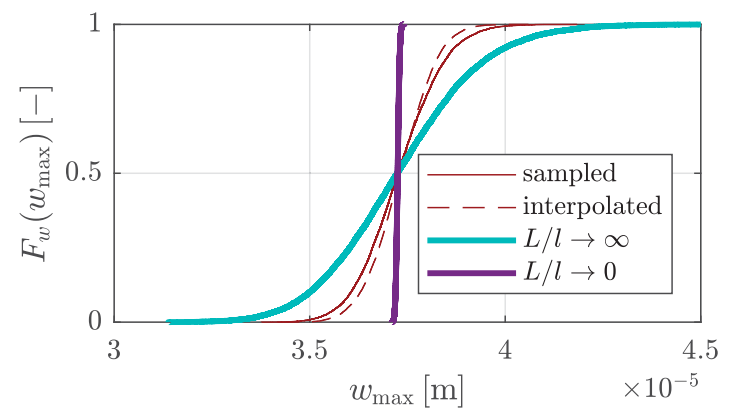

(b) $L_{E} / l=0.1$

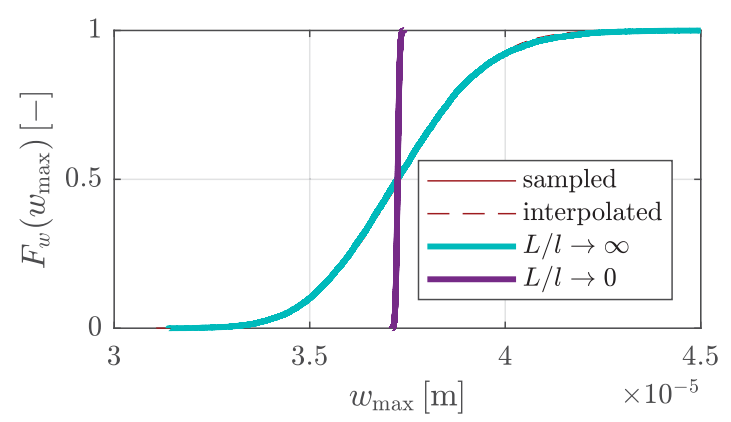

(d) $L_{E} / l=10.0$

Figure 9: Comparison of the CDFs gained by sampling and interpolation within the "absolutely no idea p-box" for different correlation lengths $L_{E} / l$ considering the Young's modulus as an imprecise random field input. 


\subsection{Investigation on the interference of two imprecise random field input parameters}

The more parameters are considered as imprecise random fields the more expensive a simulation becomes. If the underlying interval valued correlation lengths need to be discretised, each combination of $L_{i} / l$ corresponding to each parameter needs to be propagated. In this case, a cheap estimate gained by linear interpolation can still become valuable. The beam problem defined in Figure 5 is simulated again but with both parameters, the line load $q(z, \omega)$ and the Young's modulus $E(z, \omega)$, considered as imprecise random fields. The corresponding mean values $\mu_{q}$ and $\mu_{E}$ as well as standard deviations $\sigma_{q}$ and $\sigma_{E}$ are still chosen as given in Table 2 . For both parameters, the correlation length values $L^{(*)} / l=[\mathrm{WN}, 0.01,0.1,1.0,10.0, \mathrm{RV}]$ are chosen and each combination $L_{q, i} / l \times L_{E, i} / l$ is propagated. The corresponding random fields are truncated such that $\bar{\epsilon}_{i}=0.8 \%$. For the propagation of each $L_{i} / l$ combination, $n_{\mathrm{MC}}=30000$ samples are generated.

The resulting CDFs for the quantity of interest $w_{\max }$ are depicted in Figure 10. The combination of twice white noise and twice a random variable are depicted in bold lines while the vice versa combinations are depicted in bold dashed lines. For the sake of clarity, the combinations where it is $L_{q, i} / l=L_{E, i} / l$ are depicted in coloured lines, all other combinations are plotted in grey dotted lines.

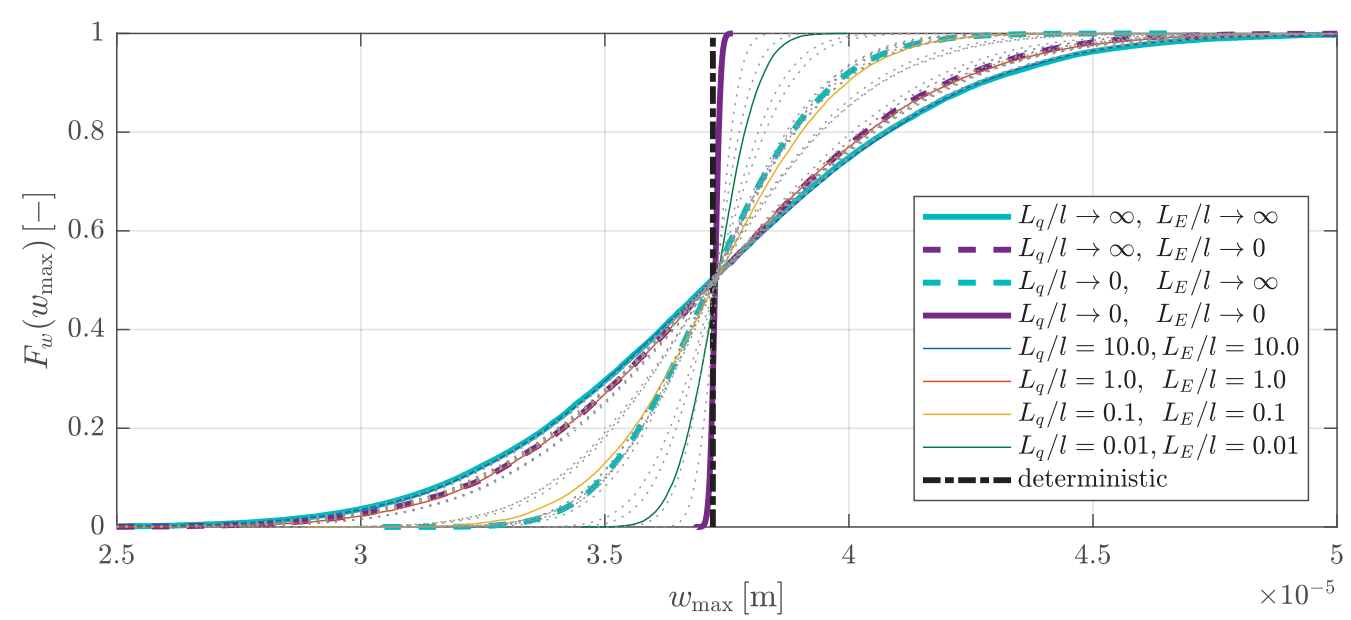

Figure 10: CDFs for different combinations of correlation length ratios $L_{q} / l$ and $L_{E} / l$ bounded by the combinations of white noise $L_{q} / l \rightarrow 0, L_{E} / l \rightarrow 0$ and random variables $L_{q} / l \rightarrow \infty, L_{E} / l \rightarrow \infty$ considering two random field input parameters.

As before, the mean value $\mu\left\{w_{\max }\right\}$ is not affected but the standard deviation $\sigma\left\{w_{\max }\right\}$. It can be seen that all $L_{i} / l$ combinations lay within the p-box defined by the white noise combination and the random field combination. Furthermore, the former seems again to converge towards the deterministic result. The "absolutely no idea p-box" can therefore be defined by only propagating the combination of both parameters being a random variable.

The resulting standard deviation $\sigma\left\{w_{\max }\right\}$ depending on the input combination of $\sigma\left\{\hat{\mu}_{q, j}\right\}$ and $\sigma\left\{\hat{\mu}_{E, j}\right\}$ is depicted in Figure 11. The blue surface is spanned by the results corresponding to $\left[0, \sigma\left\{w_{\max }^{\mathrm{RV}, q}\right\}\right] \times\left[0, \sigma\left\{w_{\max }^{\mathrm{RV}, E}\right\}\right]$. The results $\sigma\left\{w_{\max }\right\}$ gained by propagating the pairs $L_{q, i} / l$, $L_{E, i} / l$ are marked by a cross, while the interpolated value corresponding to this input is marked by a dot on the surface. Furthermore, the interpolated and simulated values corresponding to each other are connected by a line. This way the distance between the simulation cross and the interpolation surface is visualised. 


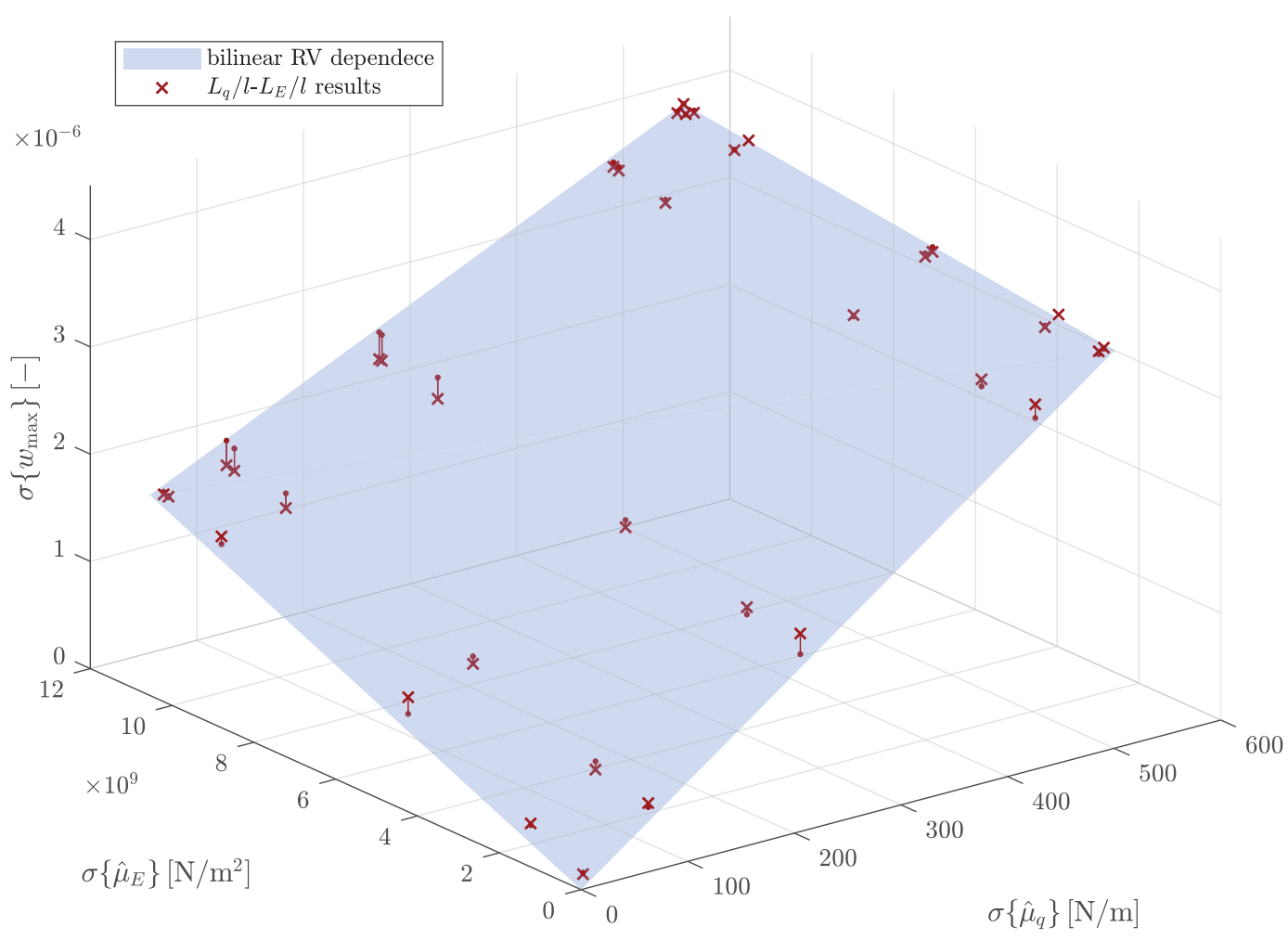

Figure 11: Dependence of the output standard deviation $\sigma\left\{w_{\max }\right\}$ on the input standard deviations $\sigma\left\{\hat{\mu}_{q, j}\right\}$ and $\sigma\left\{\hat{\mu}_{E, j}\right\}$ of the individual mean values $\hat{\mu}_{q, j}=\mu\left\{\hat{q}_{j}\right\}$ and $\hat{\mu}_{E, j}=\mu\left\{\hat{E}_{j}\right\}$, respectively, with $n_{\mathrm{MC}}=30000$ random field realisations $\hat{q}_{j}(z, \omega)$ and $\hat{E}_{j}(z, \omega)$ when two imprecise random fields are considered.

Compared to the two studies discussed in Subsection 4.1, the bilinear interpolation surface matches most of the simulation results even better. The computational cost could therefore be reduced drastically by only propagating the parameters modelled by random variables and avoiding the propagation of several correlation length combinations. Additionally, this would completely save the cost to determine the KL expansion, which can become expensive when no analytic solution is available. The cost of each individual realisation can be furthermore reduced significantly when the random property is constant for each realisation. Finally, propagating one or several random variables means a low stochastic dimension which enables more sophisticated sampling techniques than brute force MC sampling. As discussed in Subsection 3.1, the propagation of just one random field can become highly dimensional. Therefore sophisticated sampling methods often suffer from the curse of dimensionality when they are used to propagate random fields.

\section{CONCLUSION AND PERSPECTIVES}

In this contribution imprecise random fields described by interval valued correlation lengths have been investigated. In a first study, the influence of the correlation length $L$ on a standard normal distributed random field has been studied in general. To describe the variability of a random field $X(\boldsymbol{z}, \omega)$ corresponding to $L$, the mean value $\mu\left\{\hat{\mu}_{X, j}\right\}$ and standard deviation $\sigma\left\{\hat{\mu}_{X, j}\right\}$ of the individual random field realisations mean values $\hat{\mu}_{X, j}$ have been introduced. It 
could be shown that the former converges towards the mean value $\mu_{X}$ used to define the random field, while the converged value of the latter depends on $L$. However, the bounds of $\sigma\left\{\mu_{X}, j\right\}$ are defined by the limits of the correlation length, $L \rightarrow 0$ describing white noise and $L \rightarrow \infty$ standing for a random variable. For the applied linear elastic example, these limits propagate to a p-box which includes all other solutions corresponding to any $L$. As $L \in(0, \infty)$ represents all possible correlation lengths when no information is available, this p-box has been called "absolutely no idea p-box".

In a second step the dependence between input and output of the simple linear mechanical model has been investigated in terms of different imprecise random field input parameters. As the mean value of the quantity of interest $Y$ is barely affected by the correlation length, the focus has been on the standard deviation. It has been shown that the dependence between the standard deviation $\sigma\{Y\}$ of the quantity of interest and $\sigma\left\{\mu_{X, j}\right\}$ is not perfectly linear. However, determining it by assuming a linear dependence within the "absolutely no idea pbox" has shown to result in a good estimate. Furthermore, as the real standard deviation $\sigma\{Y\}$ is underestimated, a linear interpolation of the lower interval bound results in a conservative p-box, when $L \in[\tilde{L}, \infty)$ is considered. According to the fact that the correlation length (as well as the autocorrelation function itself) is usually unknown, interpolating within an "absolutely no idea p-box" can be a computational cheap method in terms of engineering application. As the white noise converges towards a vertical line of the mean value $\mu\{Y\}$ corresponding to the random field, only the random variable needs to propagated to determine this limit representation of the p-box, avoiding the need for computationally expensive random field discretisation. By that, the stochastic dimensions are reduced drastically and perhaps, more efficient low dimensional sampling schemes could be applied to further reduce the computational cost for engineering analysis.

For engineering applications the suggested approach appears very attractive. Further investigations are needed to investigate nonlinear problems. Here, for some parameters the dependence between input and output can become more complex. Still, a first linear estimate can be used to reduce the sampling effort.

\section{REFERENCES}

[1] M. Beer, S. Ferson and V. Kreinovich, Imprecise probabilities in engineering analyses. Mech. Syst. Signal Pr. 37. 4-29, 2013.

[2] W. Betz, I. Papaioannou, D. Straub, Numerical methods for the discretization of random fields by means of the Karhunen-Loève expansion. Comput. Methods Appl. Mech. Engrg. 271, 109-129, 2014

[3] M.M. Dannert, R.M.N. Fleury, A. Fau and U. Nackenhorst, Non-linear Finite Element Analysis under Mixed Epistemic and Aleatory Uncertain Random Field Input. M. Beer and E. Zio eds. Proceedings of the 29th European Safety and Reliability Conference (ESREL), Hanover, Germany, Septermber 22-26, 2019.

[4] M.M. Dannert, M.G.R Faes, R.M.N. Fleury, A. Fau, U. Nackenhorst and D. Moens, Imprecise random field analysis for non-linear concrete damage analysis. Mech. Syst. Signal Pr. 150, 107343, 2021

[5] A. Der Kiureghian and O. Ditlevsen, Aleatory or epistemic? Does it matter? Struct. Saf. 31 (2), 105-112, 2009 
[6] M. Faes and D. Moens, Imprecise random field analysis with parametrized kernel functions. Mech. Syst. Signal Pr. 134, 106334, 2019.

[7] R.G. Ghanem and P.D. Spanos, Stochastic Finite Elements: A Spectral Approach. New York: Springer, 1991.

[8] M. Hanss, Applied Fuzzy Arithmetic: An Introduction with Engineering Applications, Springer, 2005

[9] R. Moore, R. Kearfott and M. Cloud, Introduction to Interval Analysis, Society for Industrial and Applied Mechanics, 2009

[10] F.N. Schietzold, A. Schmidt, M.M. Dannert, A. Fau, R.M.N. Fleury,W. Graf, M. Kaliske, C. Könke, T. Lahmer and U. Nackenhorst, Developement of fuzzy probability based random fields for the numerical structural design. GAMM-Mitteilungen 42, e201900004, 2019.

[11] B. Sudret and A. Der Kiureghian, Stochastic finite element methods and reliability - A state-of-the-art report, Tech. rep., report no. UCB/SEMM-2000/08, Department of Civil \& Environmental Engineering, University of California, Berkeley, 2000. 